\title{
Cu(II) Complex of L-Leucine Favor a Different Type of Crystal Structure from Cu(II)-L-Val and Cu(II)-L-Ile
}

\author{
Masaaki Yokota1 $^{*}$, Shingo Kikuchi' ${ }^{1}$ Jun Sen ${ }^{2}$, Toshimichi Kamei ${ }^{2}$, Norihito Doki ${ }^{1}$ \\ ${ }^{1}$ Department of Chemistry and Bioengineering, Iwate University, Morioka, Japan \\ ${ }^{2}$ Research Institute for Bioscience Products \& Fine Chemicals, Ajinomono Co., Inc., Kawasaki, Japan \\ Email: *myokota@iwate-u.ac.jp
}

Received 1 December 2015; accepted 17 January 2016; published 20 January 2016

Copyright (C) 2016 by authors and Scientific Research Publishing Inc.

This work is licensed under the Creative Commons Attribution International License (CC BY). http://creativecommons.org/licenses/by/4.0/

c) (i) Open Access

\begin{abstract}
Crystallization behavior of $\mathrm{Cu}$ (II) complexes of branched chain amino acids (BCAAs) has been studied. Mixing of $\mathrm{Cu}$ (II) and L-leucine in solution caused immediate precipitation of $\mathrm{Cu}$ (II)-L-Leu complex, while no precipitants were obtained when L-Val or L-Ile were used as ligands. These results are discussed based on structure differences among the precipitants.
\end{abstract}

\section{Keywords}

Crystallization, Crystal Structure, Copper Complex, Branched Chain Amino Acid

\section{Introduction}

It is well known that amino acids can act as ligands of metal cations and it is described even in the basic chemistry textbooks. Among the long history of the metal-amino acid complexes, Cu(II)-amino acids have been widely studied and have been applied for various purpose such as chiral separators [1] [2]. As fundamental researches, interests have been paid for their structures in gas, liquid and solid state. Usually, one Cu(II) cation binds two amino acid molecules through $\mathrm{Cu}(\mathrm{II})$-carbonyl oxygen and $\mathrm{Cu}(\mathrm{II})$-amino nitrogen bonds. $\mathrm{Cu}(\mathrm{II}) \mathrm{com}-$ plexes of amino acids form two geometric (cis/trans) isomers as shown in Figure 1. In cis-isomer (Figure 1(a)), two ligands, e.g., two carbonyl oxygen, are adjacent to each other, while two oxygen atoms are on opposite sides of the central $\mathrm{Cu}(\mathrm{II})$ cation in trans-isomer.

Our research group have been studied crystal structures of branched chain amino acid (BCAA) such as L-valine, L-leucine and L-isoleucine. In single system, the BCAAs form similar crystal structures with hydrophobic ${ }^{*}$ Corresponding author.

How to cite this paper: Yokota, M., Kikuchi, S., Sen, J., Kamei, T. and Doki, N. (2016) Cu(II) Complex of L-Leucine Favor a Different Type of Crystal Structure from Cu(II)-L-Val and Cu(II)-L-lle. Advances in Chemical Engineering and Science, 6, 62-66. http://dx.doi.org/10.4236/aces.2016.61007 


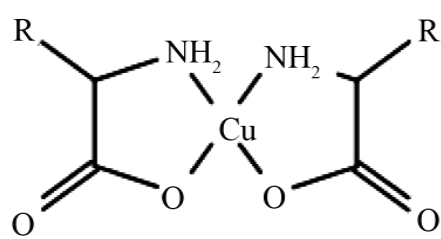

(a)

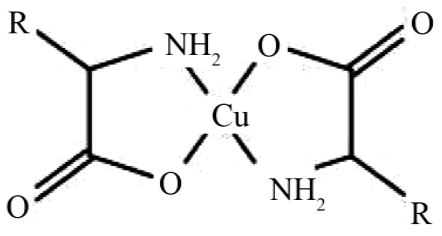

(b)

Figure 1. Moclecular structures of (a) cis- and (b) trans-Cu(II) complexes of an amino acid.

and hydrophilic double layers. This similarity in crystal structures brings some difficulty in BCAA production processes. We are very interested in breaking the structure difference among the three BCAAs. As one of the methods, we are very interested in $\mathrm{Cu}(\mathrm{II})$-BCAA complex formation because of possibility of conformational (cis and trans) polymorphism. In this study, some interesting results were found concerning the structure differences leading to different precipitation behavior.

\section{Experimental}

\subsection{Materials}

All amino acids, L-valine (L-Val), L-leucine (L-Leu), L-isoleucine (L-Ile) used in this study were pharmaceutical grade and were purchased from Ajinomoto Co. Ltd. As $\mathrm{Cu}(\mathrm{II})$ cation source, copper acetate, $\mathrm{Cu}(\mathrm{OAc})_{2} \cdot \mathrm{H}_{2} \mathrm{O}$ (CuOAc, Wako Chemical Co.) was used. These materials were used without purification.

\subsection{Method}

One of BCAAs (L-Val, L-Leu, L-Ile, $0.5 \mathrm{mmol}$ ) was dissolved in distilled water $(20 \mathrm{ml})$. To the solution was added $0.25 \mathrm{mmol}$ of CuOAC as solid followed by dissolution of CuOAC to form homogeneous solution. Complex formation was occurred in the homogeneous system and changes in the solution state was observed by naked eye. In this experimental, $\mathrm{pH}$ of the solution was not controlled and the solution was allowed for stand at $298 \mathrm{~K}$. If the precipitation was occurred by only the mixing, resultant crystals were filtered and its structure was analyzed. In case of no precipitation, the solvent water was evaporated and crystallization was forced to occur. Structure of the formed crystals were determined by measuring powder XRD using RigakuRINT 2200 (CuKa: $\lambda=$ $1.5406 \AA$ ). Structure of the obtained crystals were estimated by comparing the calculated patters simulated using published crystallographic data [3]-[5].

\section{Results and Discussion}

\subsection{Precipitation Behavior of $\mathrm{Cu}(\mathrm{II})(\mathrm{BCAA})_{2}$ Complexes}

Addition and dissolution of solid CuOAc in L-Val aqueous solution caused only solution color change from colorless to blue transparent. No precipitation was confirmed at the given condition as shown in Figure 2(a). In this liquid, $\mathrm{Cu}(\mathrm{II})(\mathrm{L}-\mathrm{Val})_{2}$ complex must be formed in the liquid phase, however, the concentration of the product complex must be lower than saturated concentration, leading to no assembly of the complex molecules. Interestingly, whenthe copper was mixed with L-Leu aqueous solution, immediate precipitation of blueish powders was occurred (Figure 2(b)) and the remained solution color was changed to colorless. In case of L-Ile (Figure 2(c)), almost the same results with the case of $\mathrm{Cu}(\mathrm{II})-\mathrm{L}-\mathrm{Val}$ system were observed. As mentioned above, no precipitants were obtained for $\mathrm{Cu}(\mathrm{II})-\mathrm{L}-\mathrm{Val}$ and $\mathrm{Cu}(\mathrm{II})-\mathrm{L}-\mathrm{Ile}$ at a given experimental conditions just by mixing, thus the solvent water were evaporated to obtain reactant products in solid state for confirmation of structures of the reactant products by powder XRD measurements and the results are discussed later in Section 3.3.

\subsection{Molecular and Crystal Structures of $\mathrm{Cu}(\mathrm{II})(\mathrm{BCAA})_{2}$ Complexes}

Before examine the structures of the product crystals described in Section 3.1, let's see Cu(II)(BCAA) $)_{2}$ crystal structures using reference data [3]-[5]. $\mathrm{Cu}(\mathrm{II})(\mathrm{L}-\mathrm{Val})_{2}$ complexes form two crystal structures depending on the 


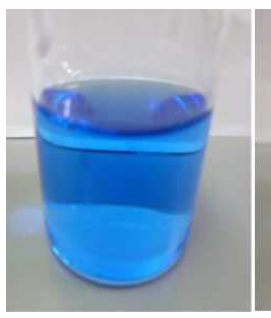

(a)

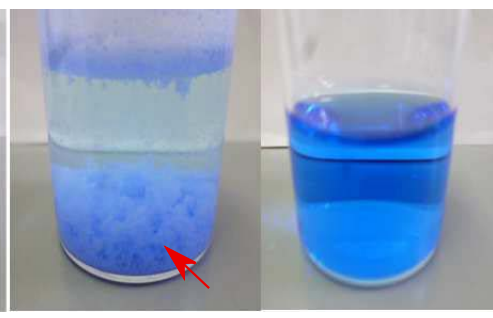

(b) (c)

Figure 2. Photographic pictures of solution after mixing CuOAc and L-BCAAs. (a) Cu(II)-L-Val, (b) Cu(II)-L-Leu, (c) $\mathrm{Cu}(\mathrm{II})-\mathrm{L}-\mathrm{Il}$. Precipitation was recognized only for $\mathrm{Cu}(\mathrm{II})$-L-Leu. Arrow in (b) indicates the precipitants.

crystallization conditions. One is hydrated form of cis-aquabis(L-valinato)copper(II), $\mathrm{Cu}(\mathrm{II})(\mathrm{L}-\mathrm{Val})_{2} \cdot \mathrm{H}_{2} \mathrm{O}$, and the other is trans-bis(L-valinato)copper(II), $\mathrm{Cu}(\mathrm{II})(\mathrm{L}-\mathrm{Val})_{2}$. As shown in Figure 3(a), the $\mathrm{Cu}(\mathrm{II})$ ion is pentacoordinated with two carbonyl oxygen, two amino nitrogen atoms and a hydrated water oxygen atom in cis$\mathrm{Cu}(\mathrm{II})(\mathrm{L}-\mathrm{Val})_{2} \cdot \mathrm{H}_{2} \mathrm{O}$ molecule. In contrast, trans-Cu(II)(L-Val) $)_{2}$ (Figure 3(b)), forms hexacoodinated complex, in which the $\mathrm{Cu}(\mathrm{II})$ cation is coordinated with two carbonyl oxygen atoms, two amino nitrogen atoms and two carbonyl oxygen atoms of neighboring trans-Cu(II)(L-Val) $)_{2}$ molecules.

For $\mathrm{Cu}(\mathrm{II})$ complex with L-Leu, only trans-isomer, trans-bis(L-leucinato)copper(II) $\left(\mathrm{Cu}(\mathrm{II})(\mathrm{L}-\mathrm{Leu})_{2}\right)$ as shown in Figure 3(c) is reported [4]. For L-Ile, both cis- and trans-isomers, cis-aquabis(L-isoleucinato)copper(II), $\left(\mathrm{Cu}(\mathrm{II})(\mathrm{L}-\mathrm{Ile})_{2} \cdot \mathrm{H}_{2} \mathrm{O}\right)$ and trans-aquabis(L-isoleucinato)copper(II) $\left(\mathrm{Cu}(\mathrm{II})(\mathrm{L}-\mathrm{Ile})_{2} \cdot \mathrm{H}_{2} \mathrm{O}\right)$ are known to exist, however, crystal structure of only cis-isomer (Figure 3(d)) was reported [5]. From Figure 3 it can be understand, cis-isomers are hydrated and form pentacoordination structures, while trans-isomers are water-free and form hexacoodination structures.

Packing motif of these molecules in crystals are shown in Figure 4. In cis-Cu(II)(L-Val) $)_{2} \cdot \mathrm{H}_{2} \mathrm{O}$ and trans$\mathrm{Cu}(\mathrm{II})(\mathrm{L}-\mathrm{Val})_{2}$ complexes, Figure 4(a) and Figure 4(b), both of the two crystal structures have hydrophobic and hydrophilic double layers. Difference between them can be seen in intermolecular interactions. In cis-Cu(II)(L$\mathrm{Val})_{2} \cdot \mathrm{H}_{2} \mathrm{O}$, only intermolecular hydrogen bond are formed between molecules as shown by dotted lines in Figure 4(a), while $\mathrm{Cu}-\mathrm{O}$ coordination interaction are dominant intermolecular interaction in trans-isomer (Figure 4(b)). The same molecular interaction patterns are true for trans-Cu(II)(L-Leu) $)_{2}$ (Figure 4(c)) and cis-Cu(II)(LIle) $)_{2} \cdot \mathrm{H}_{2} \mathrm{O}$ (Figure 4(d)) crystals. In either cases, cis-isomer forms hydrogen bonding, while trans-isomer forms coordination interaction. From these results, trans-isomer must be more stable than cis-isomer.

\subsection{Structures of the Precipitated Cu(II)(BCAA $)_{2}$ Crystals}

In Figure 5, measured and calculated patterns of powder XRD were shown for $\mathrm{Cu}(\mathrm{II})(\mathrm{L}-\mathrm{Val})_{2}$ (Figure 3(a)), $\mathrm{Cu}(\mathrm{II})(\mathrm{L}-\mathrm{Leu})_{2}$ (Figure 3(b)), $\mathrm{Cu}(\mathrm{II})(\mathrm{L}-\mathrm{Ile})_{2}$ (Figure 3(c)), respectively. In either cases, observed XRD patterns are shown in blue lines, while red and green lines show calculated patterns using reported crystallographic data.

For $\mathrm{Cu}(\mathrm{II})(\mathrm{L}-\mathrm{Val})_{2}$ complex crystals, two crystal structures with cis- and trans-isomers respectively were reported. By comparing the measure XRD of the precipitant (blue line in Figure 5(a)) with the calculated value of cis- and trans-isomers (red and green lines, respectively), the measured blue line is almost agreed with cis-isomer. This means cis-isomer was mainly precipitated. Small peaks at around 7 is appeared which has the same peak of green (trans) line. This means small amount of trans-isomer was included in the cis-complex. This is not surprising results when looking at Figure 6. Figure 6 shows crystal structure of hydrated cis-Cu(II)(L$\mathrm{Val})_{2} \cdot \mathrm{H}_{2} \mathrm{O}$ crystal viewing along $<011>$ direction. For clarity, hydrated water oxygen atoms are shown by big red ball. Hydrophilic channels are formed in the crystal and water molecules are sandwiched among them. Since the water molecules are fixed with the L-Valine hydrophilic wall just by weak hydrogen bond. It can be expected the sandwiched water molecules are able to be released through the channel with the elevation of temperature even lower than water boiling temperature. In our crystallization experiment, hydrated cis-complex crystals were obtained, however, the water molecule must be released when the sampled crystals were allowed to in contact with open air. In case of L-Leuas ligand (Figure 5(b)), the measure XRD pattern is agreed with trans isomer (red line), while L-Ile was precipitated as cis-form. 


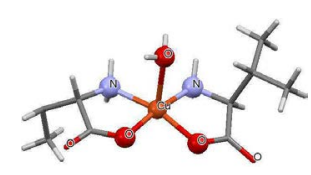

(a)

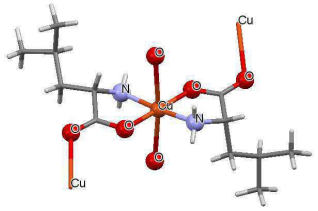

(c)

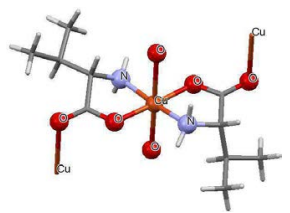

(b)

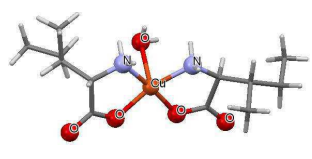

(d)

Figure 3. Molecular structures of $\mathrm{Cu}(\mathrm{II})(\mathrm{BCAA})_{2}$ in crystalline state drawn using reference crystallographic data [3]-[5]. (a) cis-Cu(II)(L-Val $)_{2} \cdot \mathrm{H}_{2} \mathrm{O}$, (b) trans-Cu(II)(L-Val) $)_{2}$, (c) trans-Cu(II)(L-Leu) $)_{2}$, (d) cis-Cu(II)(L-Ile $)_{2} \cdot \mathrm{H}_{2} \mathrm{O}$. In the model, orange $=$ copper, blue $=$ nitrogen, red $=$ oxygen, gray $=$ carbon, white $=$ hydrogen .

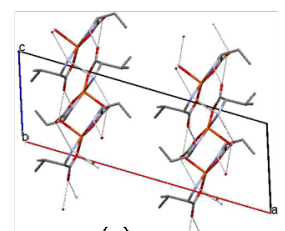

(a)

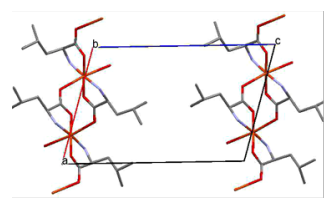

(c)

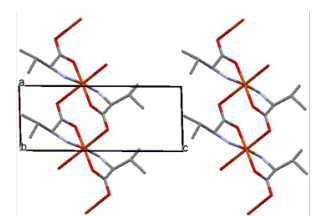

(b)

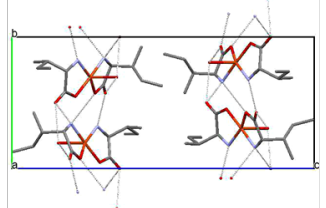

(d)

Figure 4. Crystal structures of $\mathrm{Cu}(\mathrm{II})(\mathrm{BCAA})_{2}$ drawn using reference crystallographic data [3]-[5]. (a) cis-Cu(II)(L-Val) $)_{2} \cdot \mathrm{H}_{2} \mathrm{O}$, (b) trans-Cu(II)(L-Val) $)_{2}$, (c) trans-Cu(II)(L-Leu $)_{2}$, (d) cis-Cu(II)(L-Ile $)_{2} \cdot \mathrm{H}_{2} \mathrm{O}$.

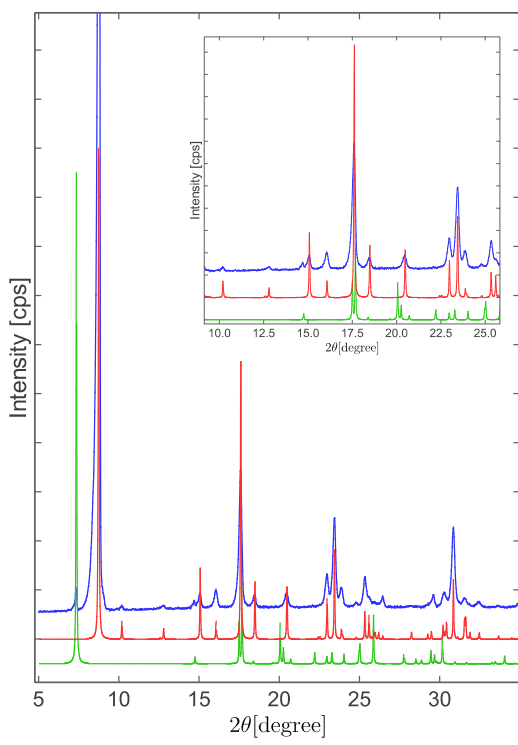

(a)

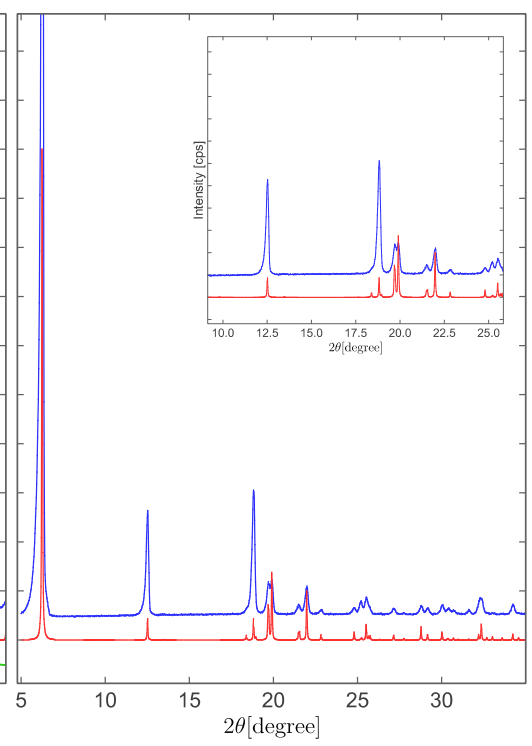

(b)

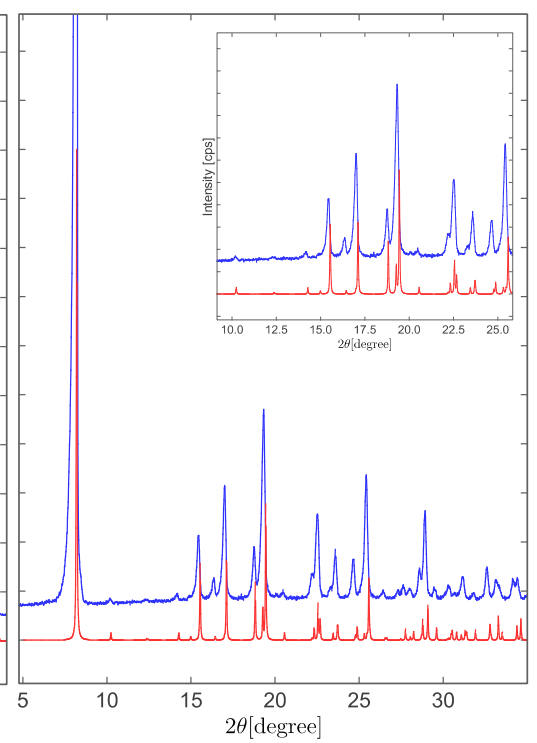

(c)

Figure 5. Powder XRD patterns of (a) $\mathrm{Cu}(\mathrm{II})(\mathrm{L}-\mathrm{Val})_{2}$, (b) $\mathrm{Cu}(\mathrm{II})(\mathrm{L}-\mathrm{Val})_{2}$, (c) $\mathrm{Cu}(\mathrm{II})(\mathrm{L}-\mathrm{Leu})_{2}$. Blue line: measured data. Red and green lines: calculated patterns. (a) Red: cis-isomer, Green: trans-isomer, (b) Red: trans isomer, (c) Red: cisform. 


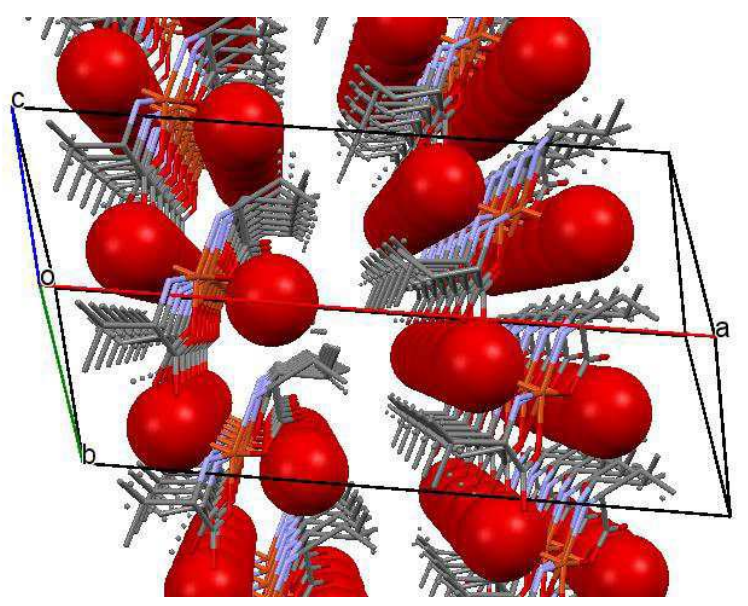

Figure 6. Formation of hydrophilic channel in which developed along $\{101\}$ direction water molecule (Red ball) are sandwiched.

Now, it may be concluded that only $\mathrm{Cu}(\mathrm{II})(\mathrm{L}-\mathrm{Leu})_{2}$ was precipitated as stable trans-form, while the others were formed metastable cis-forms. Why only L-Leu favor the trans-isomer. This is very interesting results because the three BCAAs used as ligands have similar molecular structures. Our guess for these results are as follows: Metastable cis-forms are kinetic products, while the stable trans-forms are thermodynamic products. In $\mathrm{Cu}(\mathrm{II})(\mathrm{BCAA})_{2}$ crystals formation, the metastable forms must appear first, then the metastable forms transform into stable forms. In this transformation process, side chain effect of the BCAAs must appear.

\section{Conclusion}

$\mathrm{Cu}(\mathrm{II})$ complex formation of L-Leucine leads to self-precipitation only by mixing the two reactants in the solution. On the other hand, no self-precipitation occurred when L-Val and L-Ile were used as ligands. These results may be explained only L-Leu favor stable trans conformation rather than unstable hydrated cis conformation. Using the phenomena found in this study, separation of BCAAs, which is difficult by usual crystallization, is expected.

\section{References}

[1] Shiraiwa, T., Fukuoka, H., Yoshida, M. and Kurokawa, H. (1984) Optical Resolution of DL-Alanine by Formation of Ternary Copper(II) Complexes with L-Isoleucine. Bulletin of the Chemical Society of Japan, 57, 1675-1676. http://dx.doi.org/10.1246/bcsj.57.1675

[2] Harada, K. and Tso, W.-W. (1972) Optical Resolution of Aspartic Acid by Using Copper Complexes of Optically Active Amino Acids. Bulletin of the Chemical Society of Japan, 45, 2859-2862. http://dx.doi.org/10.1246/bcsj.45.2859

[3] Markovic, M., Judas, N. and Sabolovic, J. (2011) Combined Experimental and Computational Study of cis-Trans Isomerism in Bis(L-Valinato)Copper(II). Inorganic Chemistry, 50, 3632-3644. http://dx.doi.org/10.1021/ic102585f

[4] Fawcett, T.G., Ushay, M., Rose, J.P., Lalancette, R.A., Potenza, J.A. and Schugar, H.J. (1979) Molecular Structures of the Copper-Amino Acid Complexes Bis(L-Leucinato)Copper(II) and Bis(D,L-2-Aminobutyrato)Copper(II). Inorganic Chemistry, 18, 327-332. http://dx.doi.org/10.1021/ic50192a024

[5] Weeks, C.M., Cooper, S. and Norton, D.A. (1969) The Crystal Structure of the Copper(II) Complex of L-Isoleucine. Acta Crystallographica, B25, 443-450. http://dx.doi.org/10.1107/S0567740869002408 